\title{
A COMPREHENSIVE REVIEW ON GENETICS AND ITS IMPACT ON HUMAN DISEASES
}

\author{
Prachi Kalra \\ Amity Institute of Biotechnology \\ Amity University, Noida, India
}

\begin{abstract}
In 1866, when Mendel was carrying out his pea plant experiments in the monastery, nobody could predict what was to come. A genius whose ideas were appreciated after 34 years of their discovery, quite literally planted the seeds for the genetics that we're familiar with, today. The important breakthrough was the completion of The Human Genome Project in 2003, which meant that scientists now had the genetic dictionary in their hands for an in-depth review of DNA and associated mutations. Eventually, the picture started to become clearer and our earlier speculation about the role of family history and environment in genetic disorders, now had concrete proof.
\end{abstract}

The modern-day geneticist aims to identify, diagnose, and treat gene related conditions more efficiently than ever. This article surveys the different ways in which genetic disorders manifest themselves, their causative mutations, as well as early detection and treatment.

\section{INTRODUCTION}

Disease and its inheritance is something that has perplexed researchers for hundreds of years. Although diseases could be caused through a number of ways, genetics play a significant role in passing abnormalities down the population. Each individual acquires one complete set of chromosomes from their father, and the other from their mother. In those chromosomes are encoded thousands of gene sequences which make the individual, who they are. Along with the normal genes, certain unwanted traits are also transferred from parent to offspring; Some through simple mendelian patterns, while others through a complex gene-environment interaction. [1]

The main objective of geneticists is to find the mechanism through which these diseases permeate in families, thereafter, locating particular sites on the genomic DNA that are linked to these diseases. This approach might be tedious and costly, but if done thoroughly, will direct medical professionals to devise newer diagnostic and preventative measures. [2]

\section{TYPES}

Three types of gene-derived implications are generally seen in humans- Monogenic, Chromosomal, and Multifactorial.

Inheritance of monogenic diseases is as rare as it is severe, resulting from mutations in a single gene. Although selective pressure eliminates dominant monogenic mutations, they continue to exist in the population due to severe alterations in recessive conditions [3].

Chromosomal diseases, as the name suggests, are caused due to alterations in the organism's chromosomes. These changes are brought about by their deletion, duplication, inversion, and translocations.

However, the most life threatening human genetic disorders such as cancer, diabetes, and cardiovascular diseases are often categorized as multifactorial.

Multifactorial or complex diseases, unlike the other two categories, are caused by moderated mutations in multiple genes, affecting a single phenotypic outcome. Our environment and lifestyle greatly affect how likely we are to develop a certain disease, along with our genetic susceptibility. We might not be able to change our genetic makeup, but we can adapt to a selective environment and lifestyle choice which can delay the onset of these fatal diseases. Much research is still underway as to what exactly makes complex diseases complex, but historical data proves that environment and genes are both impalpable variables. For instance, the increased risk of fair skinned people to develop skin cancer, and alcohol-flush in people of Asian descent; all are the cases of gene-environment insult at a molecular level. 


\section{International Journal of Engineering Applied Sciences and Technology, 2021 \\ Vol. 6, Issue 5, ISSN No. 2455-2143, Pages 250-256 \\ Published Online September 2021 in IJEAST (http://www.ijeast.com)}

Complex diseases are difficult to detect, owing to their disobedience to Mendelian Laws of Inheritance. The completion of the Human Genome Project came as a relief to scientists since they can now make use of Single Nucleotide Polymorphisms as markers for disease detection. SNPs are present throughout our genome and are usually harmless, however, if an SNP is present in a gene sequence and alters the formation of a functional protein, it is likely to cause a phenotypic effect.

Statistical Data shows that an individual is more likely to develop a disease if they have both the genetic susceptibility for a disease along with environmental contamination. [4]

\section{COPY NUMBER VARIATION DISEASES}

Errors during cell division can lead to certain parts of the chromosome being more vulnerable to breakage, often resulting in mutations such as duplication, deletion and translocation in multiple genes.

Duplication occurs when extra copies of genes are formed in a particular chromosomal region. They can be Tandem or displaced duplications depending on the location of the extra sequence. Copied gene sequences mean copied proteins expressed for the same gene. Since embryonic development depends on the careful measurements of essential proteins required, altering the gene dosage could lead to developmental problems. [5]

Chromosomal deletions are caused due to the loss of DNA sequences. The amount of damage depends upon the location and size of the deleted genes. If deletions happen to span a centromere for instance, the byproduct would be a chromosome without a centromere which will be lost during cell division. Haploinsufficiency is another abnormality which is caused by deletions, where one copy of the allele is lost and so only one allele remains, which affects the gene dosage and its controlled regulation for successful expression. [6]

The aftermath of chromosomal deletions is more catastrophic than that of duplications, displaying increased phenotypic symptoms such as mental retardation, short stature, larynx malformations and other physical defects. Two of the most notable deletion diseases reported in humans are cri du chat and Prader-Willi syndrome [7]. The former is caused due to the deletions in the short arm of the 5th chromosome, whereas the latter due to deletion in the long arm of chromosome 15.

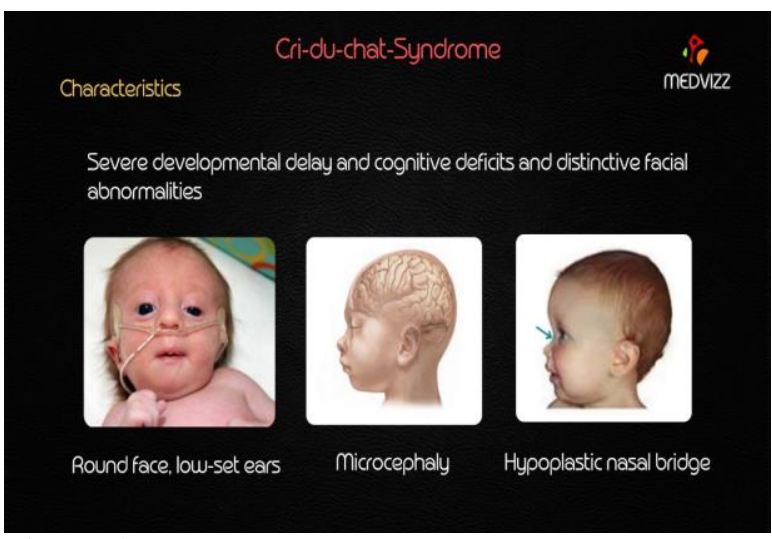

Figure 1 Cri Du Chat Syndrome

\section{BIRTH DEFECTS}

The genetic tree of congenital defects has its roots in mainly 3 domains, i.e,. chromosomal abnormalities, single gene defects, and multifactorial influencers.

Chromosomal defects are made apparent during the nuclear events of conception. However, only a few zygotes with these abnormalities are carried through to term. The ones which do manage to incubate, exhibit severe diseases such as Down Syndrome, Edward Syndrome and Patau syndrome; caused by the trisomy of chromosome number 21,18 and 13 respectively.

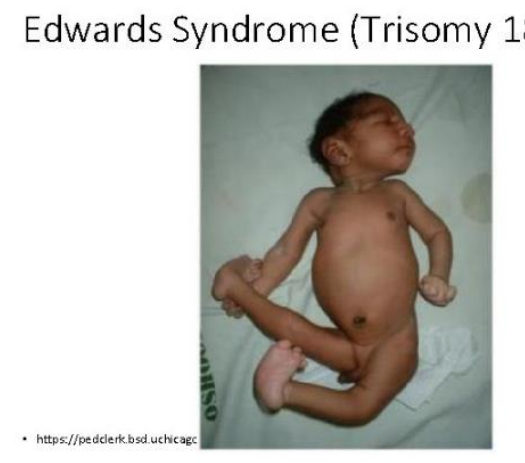

Figure 2 Edwards Syndrome

Edwards disease affects the infant's mental and physical growth, breathing and feeding, and proper functioning of the kidneys, intestines, and heart whereas Patau syndrome is associated with neurological defects, mental and motor insufficiencies, polydactyly, along with defects of the heart, eye and spine. 


\section{International Journal of Engineering Applied Sciences and Technology, 2021 \\ Vol. 6, Issue 5, ISSN No. 2455-2143, Pages 250-256 \\ Published Online September 2021 in IJEAST (http://www.ijeast.com)}

The unfortunate babies who are born with either of the conditions do not have to continue suffering from them since they die soon after their birth, owing to the complexities of these conditions. [8]

People with Down Syndrome on the other hand, do end up living a relatively long life, but not with ease. They suffer from numerous health conditions, such as heart conditions, leukemia, and Alzheimer's along with mental retardation and fertility issues in males.

Birth abnormalities caused due to single gene defects prove to be the most common inheritable diseases. One case which has had pronounced study done on it is that of phenylketonuria- a condition caused by the mutation of PAH gene responsible for producing an enzyme which in turn converts phenylalanine into tyrosine. Other inheritable mutations such as sickle cell anemia, Tay Sachs and sandhoff diseases are also common and seem to be more prevalent in specific racial and ethnic groups. Both Tay Sachs and sandhoff typically affect children, resulting in progressive neural damage which ultimately becomes their cause of death. [9]

Although many conclusions are yet to be drawn into the different aspects of multifactorial birth defects, there is surmounting evidence to believe that exposure to certain teratogens increases the chances of enduring a multitude of diseases in children who have an already predisposed genetic susceptibility to that disease.

Neural defects such as spina bifida and anencephaly for instance, have been linked with environmental factors such as folate deficiency, but also with genetic mutations in multiple genes that are responsible for the proper development of the nervous system, and trisomy of chromosome 18 .

A person's genetic makeup becomes definite at conception. It is therefore highly important that the developing fetus is exposed to a safe and healthy prenatal environment. But why? Time and time again it has been seen that mothers who are exposed to toxic agents, either voluntarily or otherwise, give birth to stillborns, premature babies, low weighted babies, mentally retarded as well as babies bearing a cleft lip and palate. Although a balanced diet and lifestyle may not ensure a healthy child, complete withdrawal from smoking and drinking during pregnancy is absolutely necessary to dismiss any risks of abnormal births.

\section{ADULT-ONSET DISORDERS}

In the 21 st century, the overall life expectancy suffered a heavy decline around the globe with thousands of seemingly healthy adults falling victim to adult-onset diseases. Although the genetic predisposition of inheriting such diseases might be present at birth, the symptoms do not manifest themselves until the later stages of life.

The most sorrowful afflictions of this kind are Alzheimer's Disease, Cancer, Huntington's disease and schizophrenia; all of which are complex diseases [10]. In order to effectively treat any of these illnesses, researchers will not only require an extensive knowledge about the multiple genes that influence their pathogenesis, but also understand the interplay between these mechanisms and the environment.

One of the reasons these diseases do not surface until much later is due to the accumulation of harmful mutations, as well as unwanted expression of harmful genes that would have otherwise stayed dormant.

In cancer patients for example, the cells are only able to become malignant after specific mutations in susceptible genes, as well as disabling a number of cancer suppressing genes. Dozens of such sequences have been identified to date, and geneticists know that the expression of some of these genes is environmentally induced. To make matters worse, alterations continue to occur even after the disease has surfaced, which eventually confers treatment resistance in the body.

Alzheimer's, which is the leading cause of dementia in the elderly, is characterized by memory loss due to progressive formation of amyloid plaques and neurofibrillary tangles in the patient's brain and nervous tissue. Recently, mutations in the APOEalong with disturbances in APP, PS1, or PS2 genesamong many other mutations in several chromosomes have been implicated in the causation of $\mathrm{AD}$, making it a polygenic trait [11].

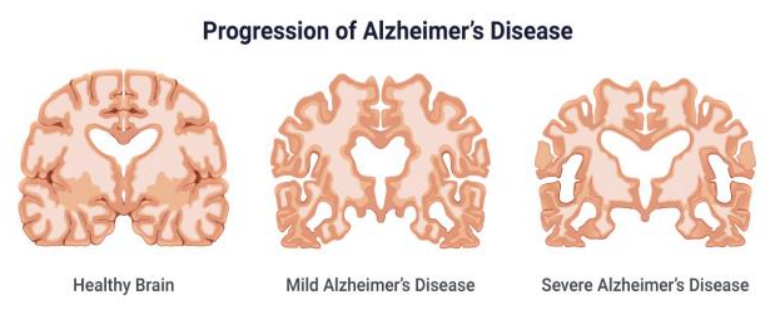

Figure 3 With severe Alzheimer's disease, brain tissue shrinks significantly. 


\section{International Journal of Engineering Applied Sciences and Technology, 2021 \\ Vol. 6, Issue 5, ISSN No. 2455-2143, Pages 250-256 \\ Published Online September 2021 in IJEAST (http://www.ijeast.com)}

Such is the case with schizophrenia, which is a chronic brain disease associated with malfunctions in the glutamate and dopamine neurotransmitter systems. Although the mode of action of this multifactorial disease, much like all others, is poorly understood, most scientists agree that there are significant underlying genetic factors to schizophrenia.

Huntington's Disease is next on the list of late-onset disorders, an inheritable disease associated with neurodegeneration. By the time the patient starts to manifest visible symptoms, they find themselves already passing the mutated HTT gene onto their progenies. Each parent affected with this condition has a 50 percent chance of birthing affected children, since $\mathrm{HD}$ is an autosomal dominant disease. Although the mutation is already present in the individual at birth, the main reason for this illness going unnoticed for so long is due to the silencing of a very important protein called brain-derived neurotrophic factor, which has significant functions in the central nervous system and is encoded by the BDNF gene [12]. The mutated Huntington gene suppresses the transcriptional activity of the latter, which results in total impairment of BDNF activity. The decreased levels of BDNF do not show any phenotypic signs for years, and it is only after the patient starts to exhibit signs of depression and subsequent cognitive impairment, that any conclusions can be drawn.

Despite achieving great success in the diagnostic and mapping part of $\mathrm{HD}$ in recent years, not much can be said about its cure- of which there is none. Although a person cannot run from this condition themself, they can certainly prevent the risk of passing it on to the next generation. A simple blood test can now tell whether or not a person is likely to suffer from $\mathrm{HD}$ through their number of CAG trinucleotide repeats.

\section{SEX LINKED DISORDERS}
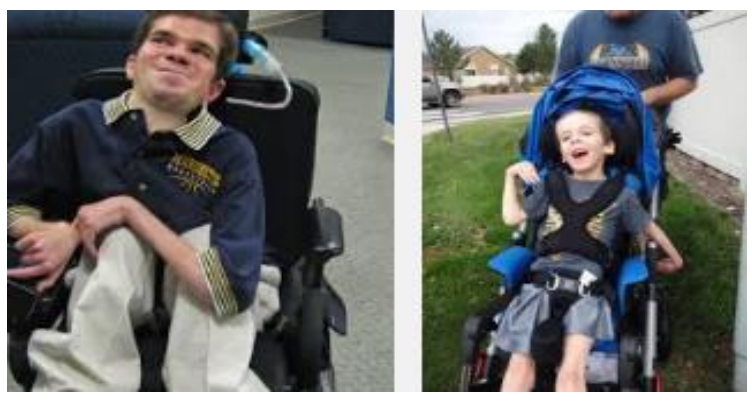

Figure 4 Duchenne muscular dystrophy
The advent of the 20th century brought with itself enormous prosperity for American Botanist Thomas Morgan and his colleagues. Among his most striking works are the insights into $\mathrm{X}$ linked Inheritance. The due diligence of Morgan in this field also put forth many interesting questions about the sex chromosomes; aberrations in which could lead to crippling, often lethal diseases.

However, through the knowledge of sex chromosome linkage also came significant developments in the diagnostic tests, done during pregnancy [13].

The most universally studied disease of this kind is one named after its founder, Duchenne muscular dystrophy, or DMD. This incurable neuromuscular condition is the result of a mutation on the $\mathrm{X}$ chromosome. It predominantly affects more males than females, because of its recessive nature. Although not affected by the disease themself, females can certainly pass on the gene onto the next generation [14]. A mutated DMD gene results in the improper functioning of dystrophin, a protein of paramount importance when it comes to maintaining muscle durability. Children affected with this disease start to show symptoms at an early age, finding immense difficulty in performing mundane tasks such as walking and running, often having to rely on mobility support for the same. The ones affected with DMD do not live past the age of 30 , the cause of death being cardiac or respiratory failure due to the progressive weakening of muscles.

\section{SOMATIC MOSAICISM}

Just like two pieces of stones making a mosaic can never be exactly identical, two cells inside the same organism can also differ from each other. This phenomenon of cell distinction is termed as mosaicism, and can arise from spontaneous DNA changes, overturning existing mutations, chromosomal aberrations as well as epigenetic changes [15].

Mosaicism has the ability to occur in both autosomal and germ cells. The probability of passing the altered genes increases several folds if it happens to occur in the latter, during or even after embryonic development.

Many mendelian disorders are the result of somatic mosaicism, ranging from metabolic disorders such as Tyrosinemia Type I caused by mutations in the FAH responsible for proper liver functioning; to 


\section{International Journal of Engineering Applied Sciences and Technology, 2021 \\ Vol. 6, Issue 5, ISSN No. 2455-2143, Pages 250-256 \\ Published Online September 2021 in IJEAST (http://www.ijeast.com)}

chromosomal instability disorders such as Bloom syndrome and Fanconi's Anemia.

Few of the selected disorders [16] are listed in the table below:

Non-Mendelian disorders are no stranger to somatic mosaicism either, the most devastating condition being cancer, discussed in depth in further sections.

It's common knowledge that apart from our nuclear DNA, human cells also contain mitochondrial DNA, inherited by our mothers. Each human cell contains numerous mitochondria, and each mitochondrion relies on its own, as well as nuclear genetic material to carry out its ATP synthesizing processes. Just like nuclear division, there exists a much less organised mitochondrial division. Attesting to its chaotic segregation, mtDNA is highly prone to mutations and can have profound effects on cellular metabolism and its functions.

Unwanted aberrations in mtDNA can lead to serious conditions, two of which are Leber hereditary optic neuropathy, associated with optic nerve disease; and post lingual deafness.

Alterations in the quantity and quality of chromosomes within a population causes chromosomal mosaicism. Females diagnosed with Turner's Syndrome, a condition associated with a karyotype of 44 autosomes and one X chromosome, are sometimes somatic mosaic with some cell populations with two $\mathrm{X}$ chromosomes (46, XX).

Somatic mosaicism has been blamed for most lifethreatening diseases also, such as trisomy of chromosome 8, and Rett's Syndrome. The latter is an $\mathrm{X}$ linked disorder caused by the mutation in MECP2 gene and is known to be lethal in males.

Somatic mosaicism is not all bad, though. Random X inactivation during fertilization events and epigenetic changes throughout an individual's lifetime are causative agents which give even identical twins their own uniqueness.

\section{THE GENETIC BASIS OF CANCER}

When it comes to diseases, science has left no stone unturned, however, a simple disease called cancer still appauls many. Statistics show that 1 in 2 men and 1 in 3 women have an overwhelming chance of developing cancer in their lifetime. This horrendous disease comes with layers that researchers have now picked apart extensively and are now battling an ongoing war.
However, before inquiring about our present-day treatment mechanisms, it's better to know how the disease manifests itself.

The term cancer refers to a group of diseases that share similar characteristics: unrestrained cell growth and proliferation. It's a multistep process characterised by a consecutive set of mutations that all accumulate to make a cell tumorous. [17]

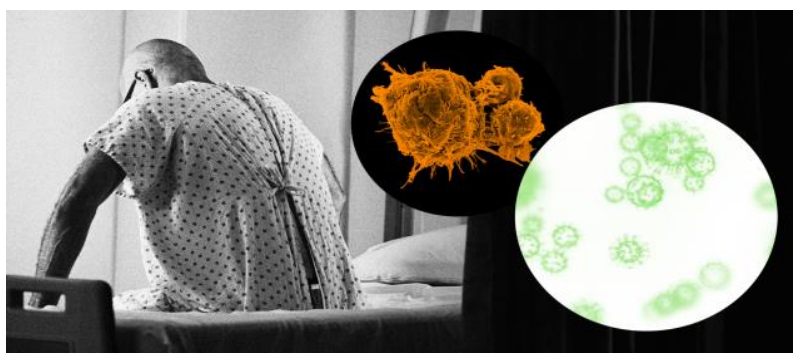

Figure 5 The fate of many cancer patients is now tied closely to the contagion's trajectory.

This clonal disease can either be inherited from one's parents, or be induced through somatic mutations. If the genetic precondition is already present in an individual, they are more likely to develop this condition and pass it on to their progenies through the germ line. [18]

For any cell to become cancerous, it will have to break some growth regulation rules. In cellular vehicles, there exist certain brakes called tumor suppressor genes, and gas pedals called proto-oncogenes, which work to inhibit and accelerate cell division, respectively. It is seen that alterations in any of these 2 very important growth regulators result in cancer. Mutations occur such that the cells acquire the superpowers to grow uncontrollably even in the presence of tumour suppressor genes or the absence of proto-oncogenes. [19]

Furthermore, cancer cells have the audacity to disobey growth regulation signals from other cells, as a virtue of which they are able to escape apoptosis and divide $\mathrm{n}$ number of times without facing any telomere (chromosomal ends) shortening.

The six hallmarks of cancer can be achieved in any pattern, resulting in the same tumorigenic capabilities at the end. Pedigree analysis as well as genetic screening of the individual with a history of cancer helps while identifying the risk and better containment at the molecular level. 


\section{International Journal of Engineering Applied Sciences and Technology, 2021 Vol. 6, Issue 5, ISSN No. 2455-2143, Pages 250-256 \\ Published Online September 2021 in IJEAST (http://www.ijeast.com)}

\section{DIAGNOSIS}

Before we pull out our therapeutic guns, it's very important to better detect the "where" and "how" of our genetic enemy. Today, diagnosis of a genetic condition requires a set of highly sophisticated methods which include a detailed history of one's family, physical examination to look for phenotypic systems, and genetic testing to analyze disease inheritance risk.

Through family history, a genetic specialist will be able to make out the frequency of occurrence of the disease, via which a genetic predisposition of developing- or perhaps being a carrier for a mutated gene- could be made apparent. If symptoms happen to occur during adulthood in more than one relative of the family, this could also stipulate a pattern of lateonset diseases in the bloodline.

Secondly, looking for slight and/or severe physical and mental deformities present since birth, such as mental retardation, slow speech, short stature, droopy eyes etc., could be indicative of a particular syndrome.

One it has been established that the patient does in fact have a genetic disorder, the next logical step is to subject them to genetic testing which will better assess doctors regarding suitable treatments, if any. These tests could be done at the cytogenetic, biochemical, and molecular level. [20]

Cytogenetic testing makes use of entire chromosomes. WBCs taken from a blood sample are cultured, fixed, stained, and then reviewed under a microscope, to study each chromosome individually. Looking for specific chromosomal breakpoints can then guide geneticists to the location of that mutated gene responsible for clinical manifestation of the disease.

Thousands of metabolic reactions in our body are mediated by multiple proteins, dressed as enzymes, hormones, promoters, and regulators. If any of these proteins fail to perform their job effectively, this could result in disease. Biochemical tests directly test proteins instead of genes. Inadequate dosage as well as mediocre or no protein activity could point to a biochemical disease.

To look for minute DNA alterations, molecular testing is the most preferable method. This kind of testing directly scans the DNA for certain mutations which are known to cause a particular disease, as in the case of cystic fibrosis. [21]

\section{TREATMENT}

In the past, doctors have been able to treat many hereditary diseases without knowing their genetic basis with great success. This was done mainly by dietary modifications, protein augmentation or through surgical processes. These therapeutic approaches are still used to this day.

For instance, if a baby is diagnosed with phenylketonuria- a disease associated with accumulation of phenylalanine in the body, they are then fed a diet low in proteins and phenylalanine as an attempt to decelerate further decline.

Hypercholesterolemia is a condition linked with high levels of bad cholesterol in the body, so the selected approach for treatment along with diet control, is administration of statins, a class of drugs tailored to regress the activity of an enzyme involved in cholesterol synthesis.

Sickle cell anemia, a condition caused by mutations in the $\beta$-subunit coding gene, is treated with hydroxyurea, a drug which increases the levels of $\gamma$ subunit in babies. These $\gamma$-subunits eventually sought to become $\beta$-subunits in adults, thereby causing lesser damage. [22]

In conditions which result in no or dysfunctional protein, a treatment called protein augmentation is carried out. In this method the proteins are added to the extracellular space, which may or may not be taken up by the cell. Protein augmentation has been deemed useful in patients with immunodeficiency, emphysema, Pompe's disease, Hemophilia, and more.

Moderate to severe genetic conditions witness the use of organ transplantation being employed frequently. Although not $100 \%$ foolproof, surgical methods for repair or replacement of diseases organs and tissues is still considered a viable option.

After the completion of human genome sequencing, a number of sophisticated therapies have now surfaced. One of which is Gene transfer, used to cure single gene recessive mutations.

It works on the principle of replacing a mutated gene with a wild type gene, thus restoring normal function. However, it's easier said than done. Clinical trials of this kind generally face problems related to a gene expression cassette, transgene expression and a delivery system. 


\section{International Journal of Engineering Applied Sciences and Technology, 2021 \\ Vol. 6, Issue 5, ISSN No. 2455-2143, Pages 250-256 \\ Published Online September 2021 in IJEAST (http://www.ijeast.com)}

The method of gene transfer surrenders in front of a dominant mutation and associated disease. Therefore, when dealing with genes of this nature, scientists prefer to silence the mRNA and thus the transcribed harmful protein. [23]

Another method, called Stem Cell Therapy, has been quoted as the modern day alternative for organ transplantation. It makes use of embryonic and somatic stem cells which are pluripotent, immortal, and capable of expressing genes corresponding to all three embryonic cell layers when induced to differentiate.

\section{CONCLUSION}

Although our accomplishments in the field of genetics are a joy to behold, we still have a lot of questions to answer. The ever-increasing curiosity of mankind might have to vanquish some ethical and social issues before it decides to test new waters. Nevertheless, our exceptional ability to pinpoint diseases on macromolecules points to our competence in developing future apparatus which will evolve our existing knowledge about genes and their impact on human diseases; and make life changing discoveries to hopefully one day free the world of these miserable illnesses.

\section{REFERENCES}

1. Bara-Jimenez, W., et al. Periodic limb movements in sleep: State-dependent excitability of the spinal flexor reflex. Neurology 54, 1609-1616 (2000)

2. Dasen, J. S., et al. A Hox regulatory network establishes motor neuron pool identity and target-muscle connectivity. Cell 123, 477 491 (2005)

3. Antonarakis, S. E., \& Beckmann, J. S. Mendelian disorders deserve more attention. Nature Reviews Genetics 7, 277-282 (2006)

4. Hunter, D. J. Gene-environment interactions in human diseases. Nature Reviews Genetics 6, 287-298 (2005)

5. Beckmann, J. S., et al. Copy number variants and genetic traits: Closer to the resolution of phenotypic to genotypic variability. Nature Reviews Genetics 8, 639-646 (2007)

6. Feuk, L., et al. Structural variation in the human genome. Nature Reviews Genetics 7 , 85-97 (2006)

7. Check, E. Patchwork people. Nature 437, 1084-1086 (2005)
8. Edwards, J. H., et al. A new trisomic syndrome. Lancet 1, 787-790 (1960)

9. Patau, K., et al. Multiple congenital anomaly caused by an extra autosome. Lancet 1, 790793 (1960)

10. Wright, A., et al. A polygenic basis for lateonset disease. Trends in Genetics 19, 97-105 (2003)

11. Kirkwood, T. A systematic look at an old problem. Nature 451, 644-647 (2008)

12. Palfi, S., \& Jarraya, B. Huntington's disease lends a hand. Nature 453, 863 (2008)

13. Casci, T. Reproductive technologies: A longterm cost. Nature Reviews Genetics 2, 489 (2001)

14. Hastings, I. M. Reproductive compensation and human genetic disease. Genetic Research 77, 277-283 (2001)

15. Armstrong, J., et al. Classic Rett syndrome in a boy as a result of somatic mosaicism for a MECP2 mutation. Annals of Neurology 50, 692 (2001)

16. Karadima, G., et al. Origin of nondisjunction in trisomy 8 and trisomy 8 mosaicism. European Journal of Human Genetics 6, 432-438 (1998)

17. American Cancer Society. Cancer Facts and Figures 2014.

18. Balmain, A. Cancer genetics: From Boveri and Mendel to microarrays. Nature Reviews Cancer 1, 77-82 (2001)

19. Futreal, P. A., et al. A census of human cancer genes. Nature Reviews Cancer 4, 177-183 (2004)

20. Francke, U., \& Kung, F. Sporadic bilateral retinoblastoma and 13q chromosomal deletion. Medical and Pediatric Oncology 2, 379-385 (1976)

21. Knudson, A. G., Jr., et al. Chromosomal deletion and retinoblastoma. New England Journal of Medicine 295, 1120-1123 (1976)

22. Brinkman, R. R., et al. Human monogenic disorders-A source of novel drug targets. Nature Reviews Genetics 7, 249-260 (2006)

23. Brinkman, R. R., et al. Human monogenic disorders-A source of novel drug targets. Nature Reviews Genetics 7, 249-260 (2006) 